\title{
Insights into Rational Design of New Class of Allosteric Effectors with Molecular Dynamics Markov State Models and Network Theory
}

In Sub M. Han*, Dylan Abramson, Kelly M. Thayer

College of Integrated Sciences, Wesleyan University

Email: han01@,wesleyan.edu, dambramson@wesleyan.edu, kthayer@wesleyan.edu

Wesleyan University

College of Integrative Sciences Hall-Atwater Laboratories Middletown, Connecticut 06459-0180

Phone: (860) 685-2210 Fax: (860) 685-2211 


\section{Supplementary Information}

\section{S1. MD-MSM}

A
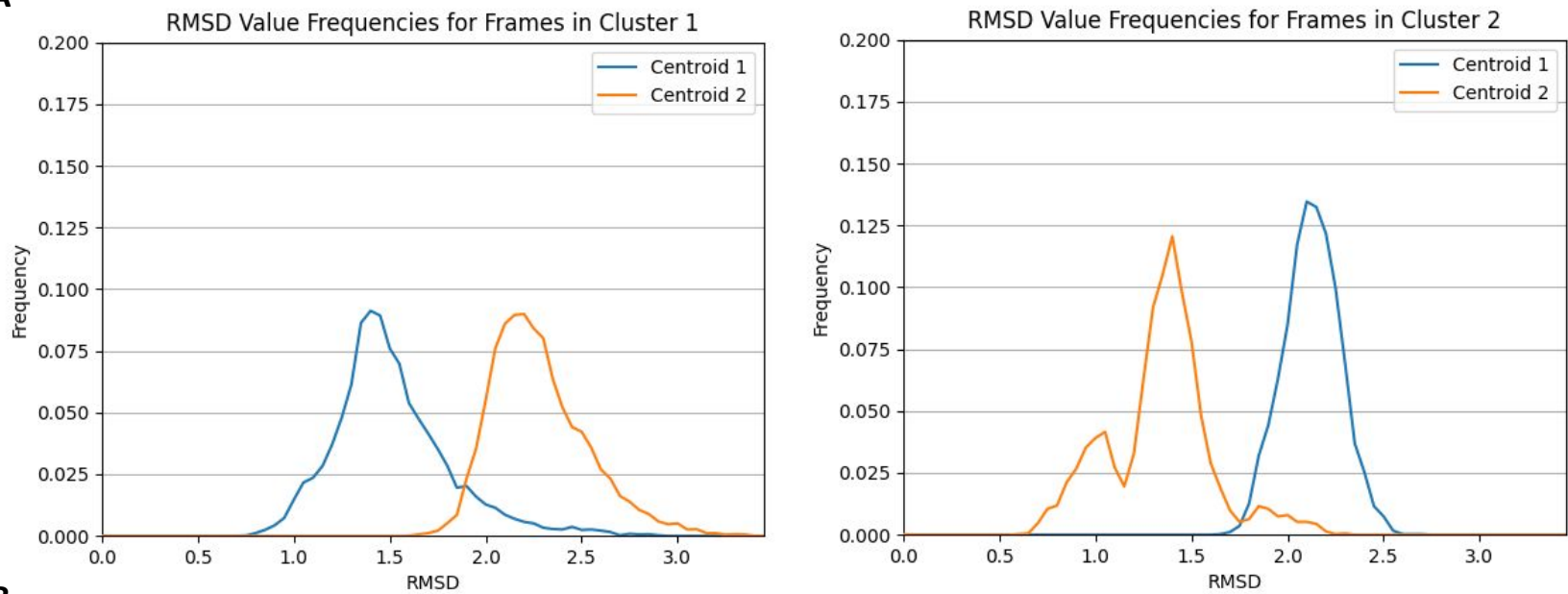

\begin{tabular}{|r|r|r|r|r|r|r|r|r|}
\hline \#Cluster & Frames & Frac & AvgDist & Stdev & Centroid & AvgCDist & Clusters & Wt_Avg_Dist \\
\hline 0 & 21401 & 0.713 & 5.029 & 1.663 & 25717 & 5.873 & 2 & 2.278586 \\
\hline 1 & 8599 & 0.287 & 3.385 & 1.207 & 18786 & 5.873 & & \\
\hline 0 & 10931 & 0.364 & 3.27 & 0.735 & 24872 & 5.678 & 3 & 1.208167667 \\
\hline 1 & 10176 & 0.339 & 4.149 & 1.23 & 4158 & 5.833 & & \\
\hline 2 & 8893 & 0.296 & 3.472 & 1.23 & 18786 & 6.348 & & \\
\hline 0 & 10562 & 0.352 & 3.173 & 0.641 & 24663 & 5.788 & 4 & 0.821336 \\
\hline 1 & 7150 & 0.238 & 3.326 & 0.725 & 5332 & 5.922 & & \\
\hline 2 & 6714 & 0.224 & 2.621 & 0.695 & 18786 & 6.563 & & \\
\hline 3 & 5574 & 0.186 & 4.246 & 1.081 & 10639 & 4.704 & & \\
\hline 0 & 10496 & 0.35 & 3.156 & 0.624 & 24663 & 5.812 & 5 & 0.6293962 \\
\hline 1 & 6982 & 0.233 & 3.272 & 0.66 & 5332 & 5.707 & & \\
\hline 2 & 6202 & 0.207 & 2.448 & 0.539 & 18786 & 6.359 & & \\
\hline 3 & 3589 & 0.12 & 3.745 & 0.776 & 10778 & 4.382 & & \\
\hline 4 & 2731 & 0.091 & 3.559 & 0.846 & 1892 & 4.98 & & \\
\hline 0 & 10398 & 0.347 & 3.137 & 0.608 & 24663 & 5.806 & 6 & 0.511483167 \\
\hline 1 & 6971 & 0.232 & 3.268 & 0.657 & 5332 & 5.816 & & \\
\hline 2 & 5535 & 0.184 & 2.267 & 0.378 & 18786 & 5.927 & & \\
\hline 3 & 2694 & 0.09 & 3.542 & 0.839 & 1892 & 4.936 & & \\
\hline 4 & 2447 & 0.082 & 3.568 & 0.715 & 11060 & 4.111 & & \\
\hline 5 & 1955 & 0.065 & 2.98 & 0.683 & 14181 & 4.571 & & \\
\hline 0 & 6905 & 0.23 & 3.252 & 0.644 & 5332 & 5.461 & 7 & 0.436622429 \\
\hline 1 & 6167 & 0.206 & 2.437 & 0.528 & 18786 & 6.604 & & \\
\hline 2 & 5778 & 0.193 & 2.879 & 0.486 & 24094 & 5.632 & & \\
\hline 3 & 4813 & 0.16 & 3.124 & 0.604 & 23992 & 4.862 & & \\
\hline 4 & 3481 & 0.116 & 3.708 & 0.765 & 10778 & 4.531 & & \\
\hline 5 & 2148 & 0.072 & 3.281 & 0.706 & 2305 & 4.431 & & \\
\hline 6 & 708 & 0.024 & 3.522 & 0.789 & 651 & 5.557 & & \\
\hline & & & & & & & & \\
\hline & & & & & & & & \\
\hline & & & & & \\
\hline & & & & \\
\hline & & & & & \\
\hline & & & & & \\
\hline
\end{tabular}

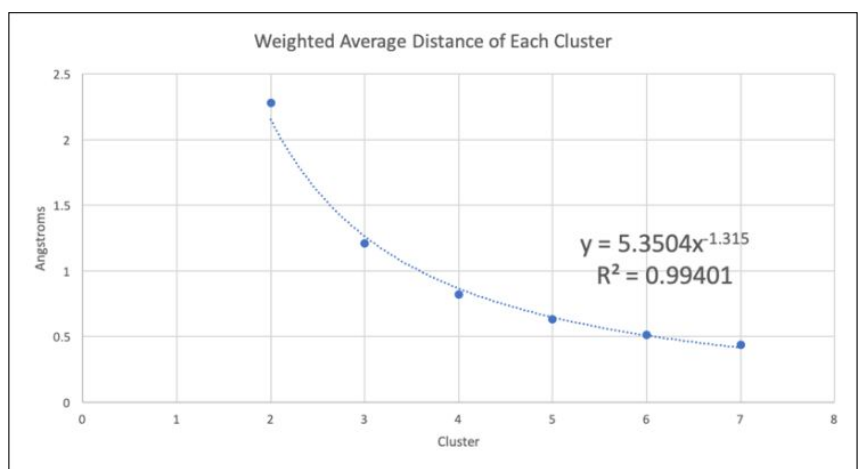

Figure S1: A. RMSD value frequencies for frames assigned to clusters 1 \& 2 . B. Weighted Average Distance of Each Cluster in table format and graphical representation of the weighted average values. 


\section{S2. Hydrogen Bonding Network}

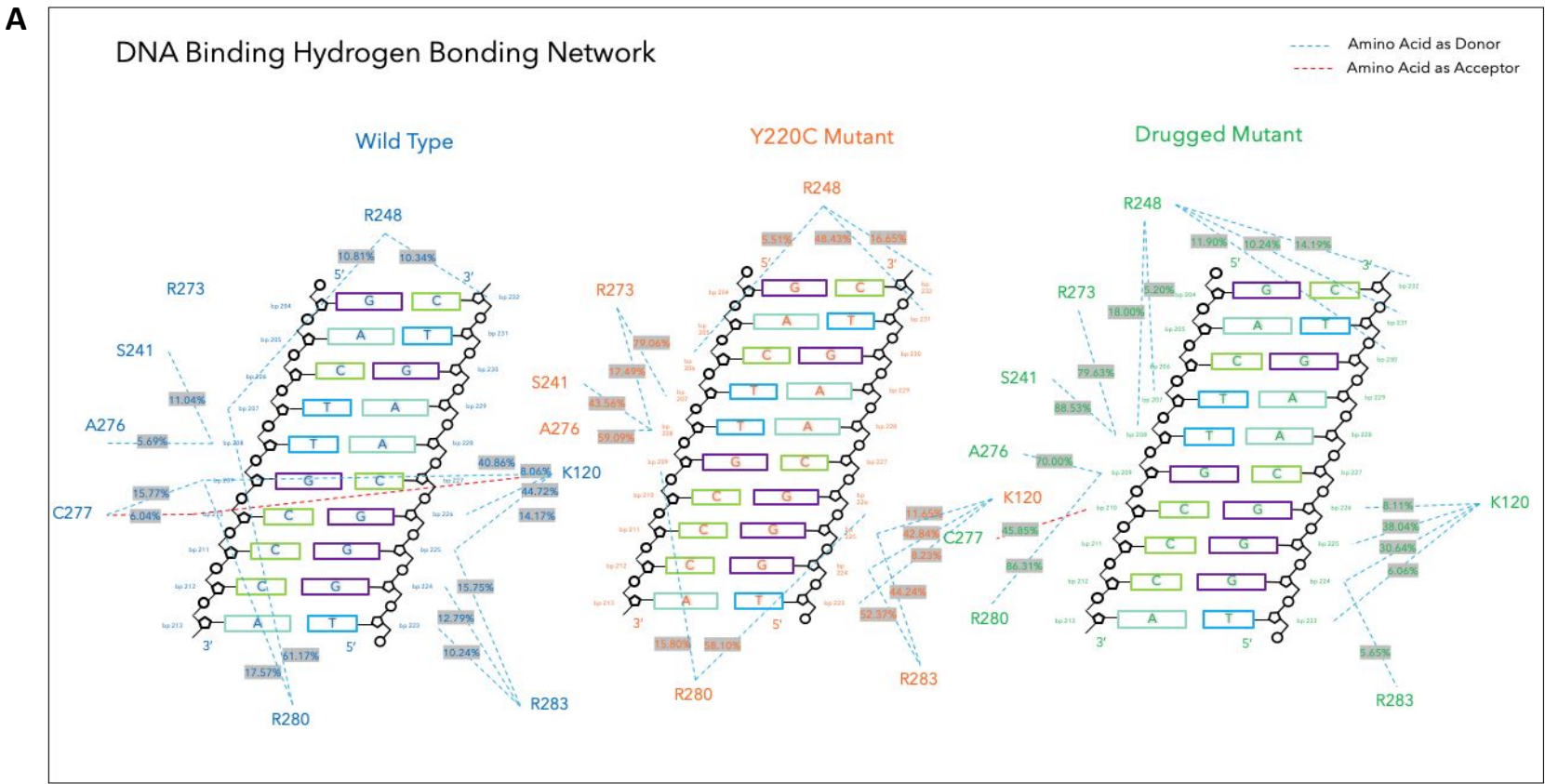

B

Number of Unique Hydrogen Bonds Between DNA and Protein

Figı

resi

Y2i

ovel

(gri

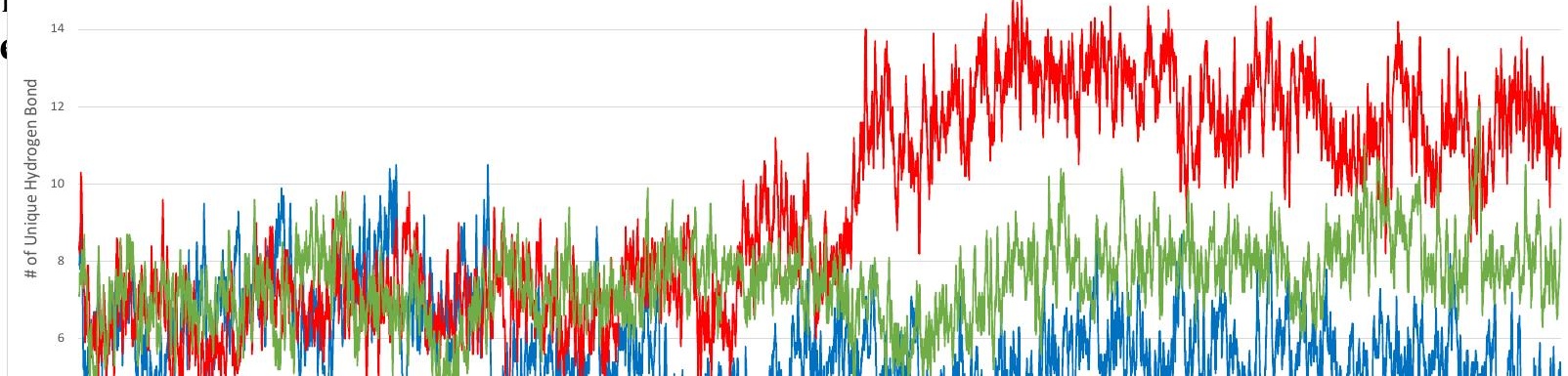




\section{S3. Data Sharing: Sample UNIX and Python Scripts}

\section{UNIX}

\section{Hydrogen Bonding Analysis Script}

parm 1tup_wt_strip_1to200.prmtop

trajin 1tup_wt_strip_1to200.trj

rms BBrsmd :1-195@C,CA,N out BBrmsd_wt.dat

hbond Backbone1 donormask :DT,DA,DG,DC acceptormask :1-195 Backbone1 out hbond1_wt.dat Backbone1 avgout avghb1_wt.dat series uuseries bbhbond1_wt.dat

go

hbond Backbone2 donormask :1-195@ acceptormask :DT,DA,DG,DC Backbone2 out hbond2_wt.dat Backbone2 avgout avghb2_wt.dat series uuseries bbhbond2_wt.dat go

\#hbond Backbone1 donormask :DT,DA,DG,DC@C,O,N,H acceptormask :1-195@C,O,N,H out hbond_wt.dat avgout avghb_wt.dat series uuseries bbhbond_wt.gn

\#hbond Backbone2 donormask :1-195@C,O,N,H acceptormask :DT,DA,DG,DC@C,O,N,H

\section{CPPTRAJ clustering script}

\#!/bin/bash

\# This script is designed for running MD-MSMs with Amber 14

\# It performs cluster analysis using AMBER'S cpptraj, using k-means

\# based on RMSD distance. Because the clustering is k-means, the number of

\# clusters is an input. Thus we try a range of number of clusters, and

\# decide which is most appropriate by metrics determining how close the

\# points are to their respective centroids.

\# Full Path:

\# /home33/\{USER\}/\{PROTEIN\}/\{CLUSTERING\}/

if [ \$\# -ne 7 ]; then

echo

echo Please enter the correct number of commandline arguments

echo

echo "Sample Input"

echo

echo './10_kcluster_master.sh' /home33/ihan01/MINDSTORE_DATA/DnD/MDMSM/ p53 wt 1192

7 strip

echo 


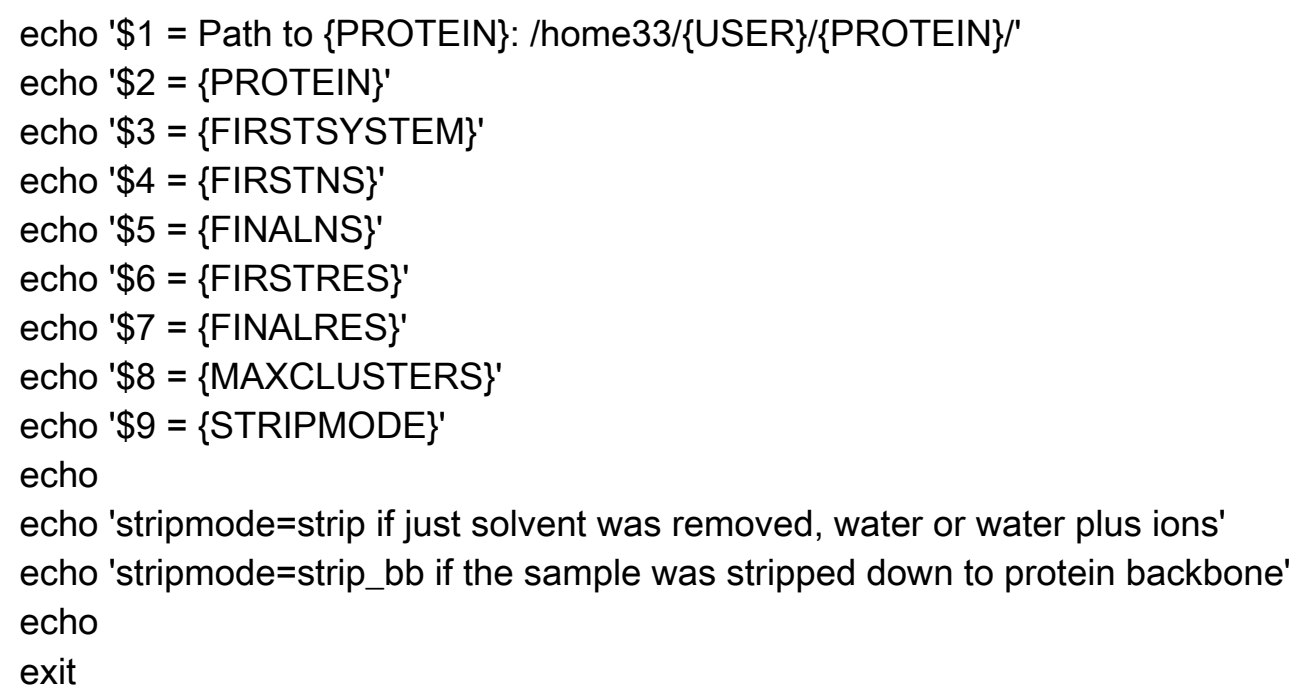

\# Step 0: Initialization and logging

PROTEINPATH $=\$ 1$

PROTEIN $=\$ 2$

FIRSTSYSTEM $=\$ 3$

\#FIRSTNS $=\$ 4$

\#FINALNS $=\$ 5$

FIRSTRES $=\$ 4$

FINALRES $=\$ 5$

MAXCLUST $=\$ 6$

STRIPMODE $=\$ 7$

\#TRAJ $=\$\{$ PROTEIN $\}$ concat_\$STRIPMODE $\} \$\{$ FIRSTNS $\}$ to $\$\{$ FINALNS $\} . t r j$

TRAJ $=\$\{$ PROTEIN $\} \_x d n a \_$half.trj

echo running 10_kcluster_master.sh $\$ 1 \$ 2 \$ 3 \$ 4 \$ 5 \$ 6 \$ 7>00 \_$README.log

\# Step 1: Generate the input file to cpptraj directing k-means clustering

for NCLUST in \$(seq $1 \$ 6)$; do

cat <<EOF> \$\{PROTEIN\}_kclust\$NCLUST-cpptraj.in

\# combined cluster analysis with cpptraj

\# load topology and both trajectories

parm \$1/wt_1ms_mdmsm.prmtop

\# reference file for aligning snapshots 
reference \$1/wt_1ms_mdmsm.inpcrd

\# load stripped trajectory file

trajin \$1/p53_dnd_1ms_short.trj

\# align all the snapshots on the reference structure

rms reference $\$ 4-\$ 5$

\# Cluster with k-means

cluster kmeans : $\$ 4-\$ 5$ mass clusters $\$$ NCLUST maxit 1000 out cluster_out averagelinkage

gracecolor summary summary_out info cluster_info repout centroid repfmt pdb clusterout cluster.nc clusterfmt netcdf

EOF

done

\# Step 2: Write the batchfile to run the clustering

cat $<<$ EOF> 11_run_\$\{PROTEIN\}_cluster_cpptraj.sh

\#!/bin/bash

\#BSUB -q amber128

\#BSUB - $n 1$

\#BSUB -J \$\{PROTEIN\}_cluster

\#BSUB -O out

\#BSUB -e err

\# commands to run calculations in Amber 14

\# cuda 8 \& mpich ${ }^{*} N E W^{*}$ for AMBER16

export PATH=/usr/local/cuda/bin:I\$PATH

export LD_LIBRARY_PATH=/usr/local/cuda/lib64:I\$LD_LIBRARY_PATH

export PATH=/usr/local/mpich-3.1.4/bin:/home/apps/amber/12cpu-only/bin:I\$PATH

export LD_LIBRARY_PATH=/usr/local/mpich-3.1.4/lib:I\$LD_LIBRARY_PATH

\# unique job scratch dirs *NEW* for AMBER16

MYSANSCRATCH=/sanscratch/

MYLOCALSCRATCH=/localscratch/

export MYSANSCRATCH MYLOCALSCRATCH

\#\# AMBER we need to recreate env, /home/apps/amber/12cpu-only is already set export PATH=/share/apps/CENTOS6/python/2.7.9/bin:IPATH

export LD_LIBRARY_PATH=/share/apps/CENTOS6/python/2.7.9/lib:ILD_LIBRARY_PATH

source /usr/local/amber16/amber.sh 


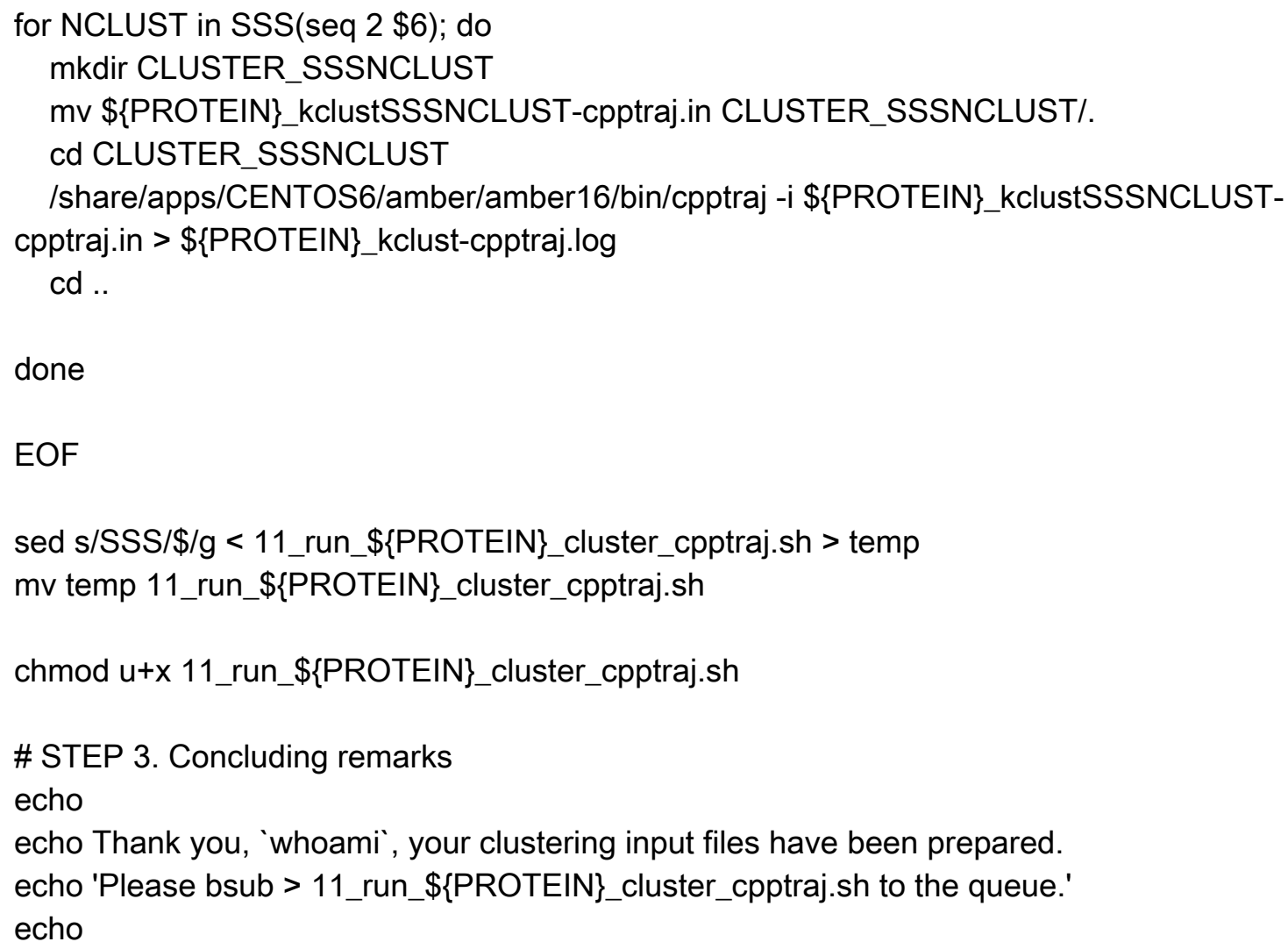

Python

\section{SECTORS}

\{

"cells": [

\{

"cell_type": "markdown", "metadata": \{\},

"source": [

"\#\#<u>Running MD Sectors on the p53 Protein $<\mathrm{u} />\mid \mathrm{n}$ ",

" "

]

\} ,

\{

"cell_type": "markdown",

"metadata": \{\},

"source": [

"**Summary:** This notebook implements the steps of Molecular Dynamics Sectors (MD Sectors), using the p53 protein as an example. The script is based off the SCA6.0 example script developed by the Ranganathan lab, as well as the previous iteration of MD Sectors from the Thayer lab with significant contributions from B. Lakhani. In", 
"In",

"**1) Information on Protein Residues. ${ }^{* *} \mid n "$,

"Import, parse and calculate the relevant data. Check how many residues are being examined. $\ln "$,

"In",

"**2) First-order statistics: position-specific Vpica Values. ${ }^{* \star} \mid n "$,

"Next, we examine overall positional vpica values for the protein. In",

"In",

"**3) Second-order statistics: conserved correlations. ${ }^{* *} \mid \mathrm{n} "$,

"Plots of the SCA matrix (\$IItilde\{C_\{ij\}\}), the associated eigenvectors and eigenspectrum, and the corresponding independent components (IC). We choose the number of significant eigenmodes, $\$ \mathrm{k}^{\wedge *}=4 \$$, by comparison of the eigenspectrum of $\$$ Iltilde $\left\{C_{-}\{i j\}\right\}$ to that for a collection of 10 randomized alignments. In",

"In",

"**4) Sector decomposition. ${ }^{* *} \mid n "$,

"Sector definition based on the top $\$ \mathrm{k}^{\wedge *} \$ \mathrm{ICs}$. We begin by fitting the top $\$ \mathrm{k}^{\wedge *} \$$ statistically significant ICs to a t-distribution, and then identify the amino acid positions contributing to the top five percent of the corresponding cumulative density function. We then construct a sub-matrix of \$Iltilde\{C_\{ij\}\} that contains only the selected top-scoring positions for the $\$ \mathrm{k}^{\wedge * \$} \mathrm{ICs}$, ordered by their degree of contribution to each IC. This plot is used to choose sector assignments. For the gprotein family, we define two sectors, sector 1 composed of ICs 1,2, and 3, and sector 2 composed of IC 4. Related to Figs. 4 and 5 of the main text. In",

"In",

"**5) Sequence subfamilies and the basis of sector hierarchy. ${ }^{* *} \mid n "$,

"We relate the two sectors (and underlying ICs) to the pattern of divergence between amino acid sequences. To do this, we make a mapping between sequence space and positional correlations using singular value decomposition. We see that the amino acid positions associated with IC1 and IC2 differentiate between different g-protein subclasses, suggesting that these regions might tune allosteric regulation in a subclass specific way. In",

" In",

"<br||>|n",

"In",

"Prior to running this tutorial, the core calculation scripts must be executed from the command line as follows: In",

" >> ./scaProcessMSA.py Inputs/PF00071_rd2.an -s 5P21 -c A -f 'Homo sapiens' -t -nln",

" >> ./scaCore.py Outputs/PF00071_rd2.dbln",

" >>./scaSectorID.py Outputs/PF00071_rd2.dbIn",

"In",

"Note that we supply pre-annotated alignments for all tutorial scripts *(the annotate_pfMSA step is slow, and should only be run once)*..In",

"In",

"**O.Rivoire, K.Reynolds and R.Ranganathan** $\mid n "$, "1/2015"

]

\} , 


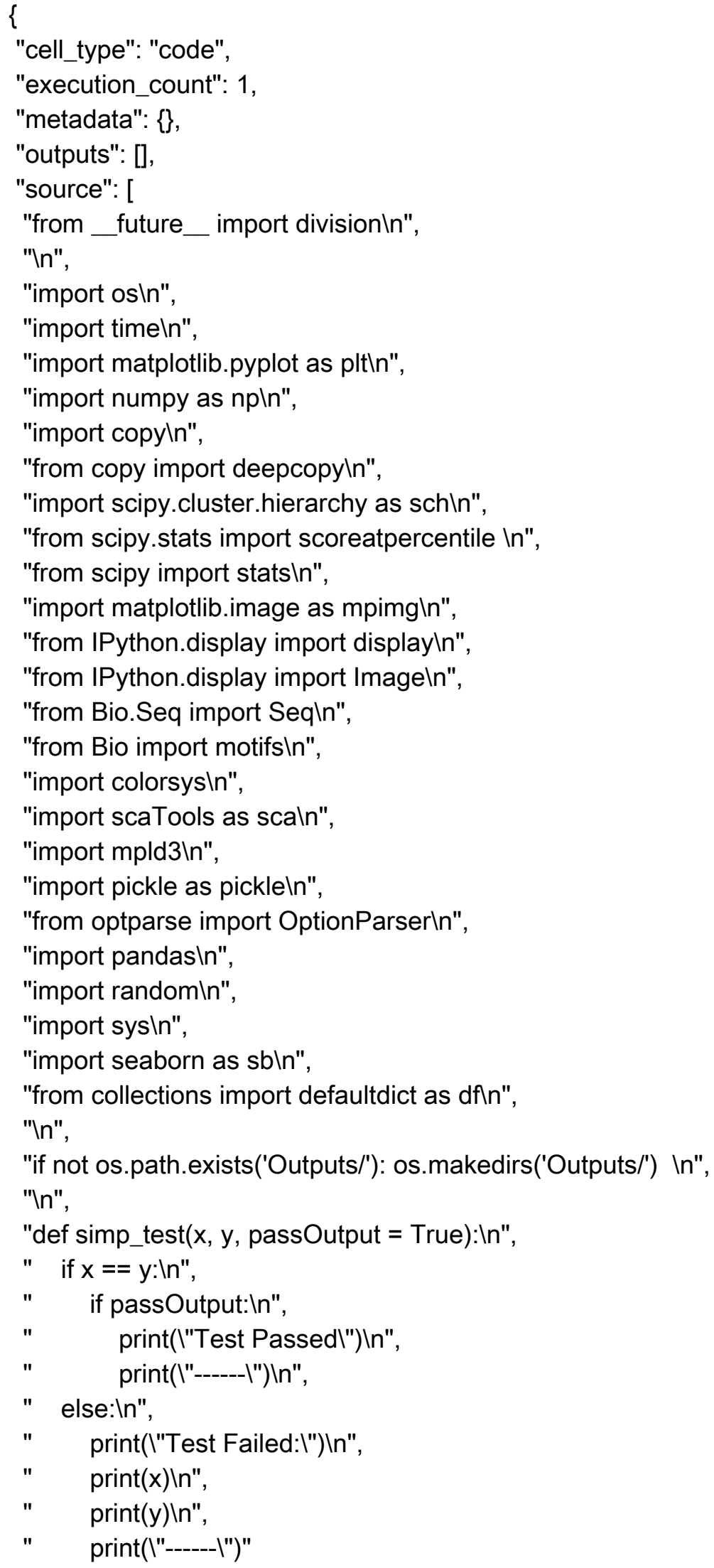




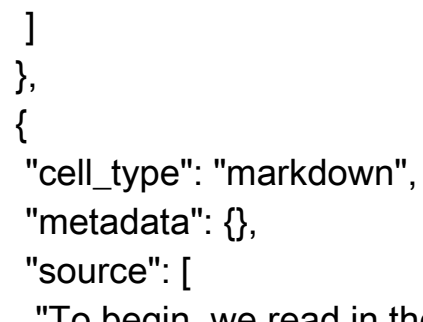

"To begin, we read in the Motion Correlation Matrix and use the SCA library to complete sector decomposition. Most information will be parsed. The following variables, however, must be hard coded to match your data: MCM_temp, amino_start and ats."

]

\} ,

\{

"cell_type": "markdown",

"metadata": \{\},

"source": [

"\#\# I. Import the Motion Correlation Matrix and Parse Relevant Data"

]

\} ,

\{

"cell_type": "code",

"execution_count": 2,

"metadata": \{\} ,

"outputs": [

\{

"name": "stdout",

"output_type": "stream",

"text": [

"There are 195 amino acids of interest. In"

]

\}

\{

"name": "stderr",

"output_type": "stream",

"text": [

"/share/apps/CENTOS6/python/3.5.2/lib/python3.5/site-packages/scipy/stats/stats.py:1713:

FutureWarning: Using a non-tuple sequence for multidimensional indexing is deprecated; use 'arr[tuple(seq)]' instead of 'arr[seq]'. In the future this will be interpreted as an array index, 'arr[np.array(seq)]', which will result either in an error or a different result.In",

" return np.add.reduce(sorted[indexer] * weights, axis=axis) / sumvalln"

]

\}

],

"source": [

"\# Get the MOTION CORRELATION MATRIXIn",

"MCM = pandas.read_excel('./Inputs/20_y220c_set_to_0.xlsx', header=None).valuesln", 


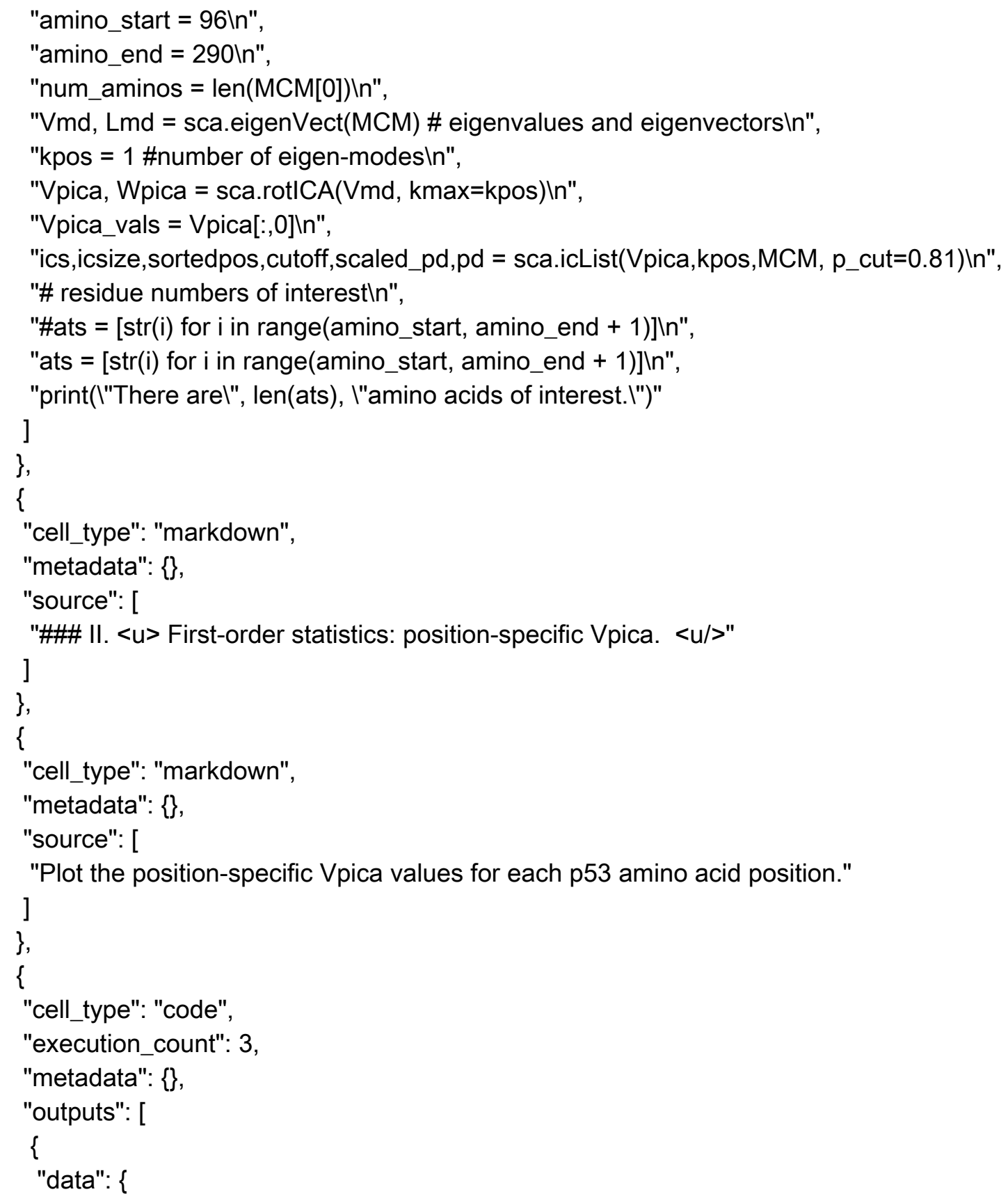

MD END: Script for python based application, Gephi for visualizing network graphs

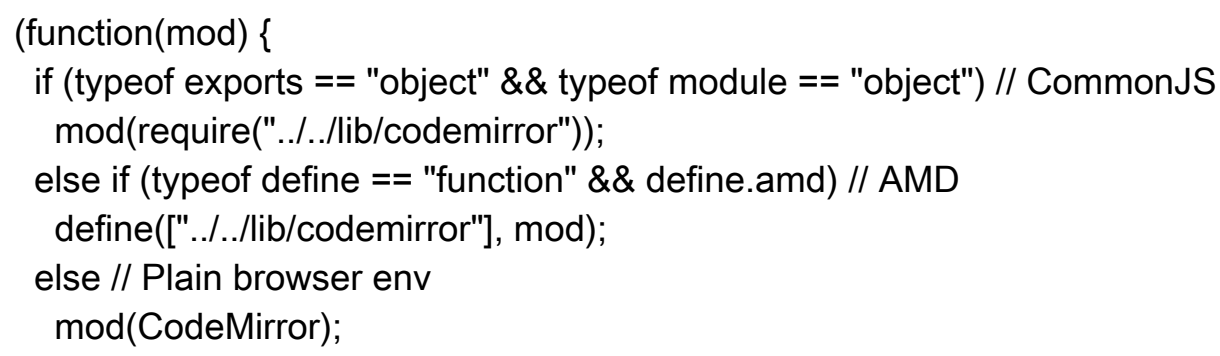




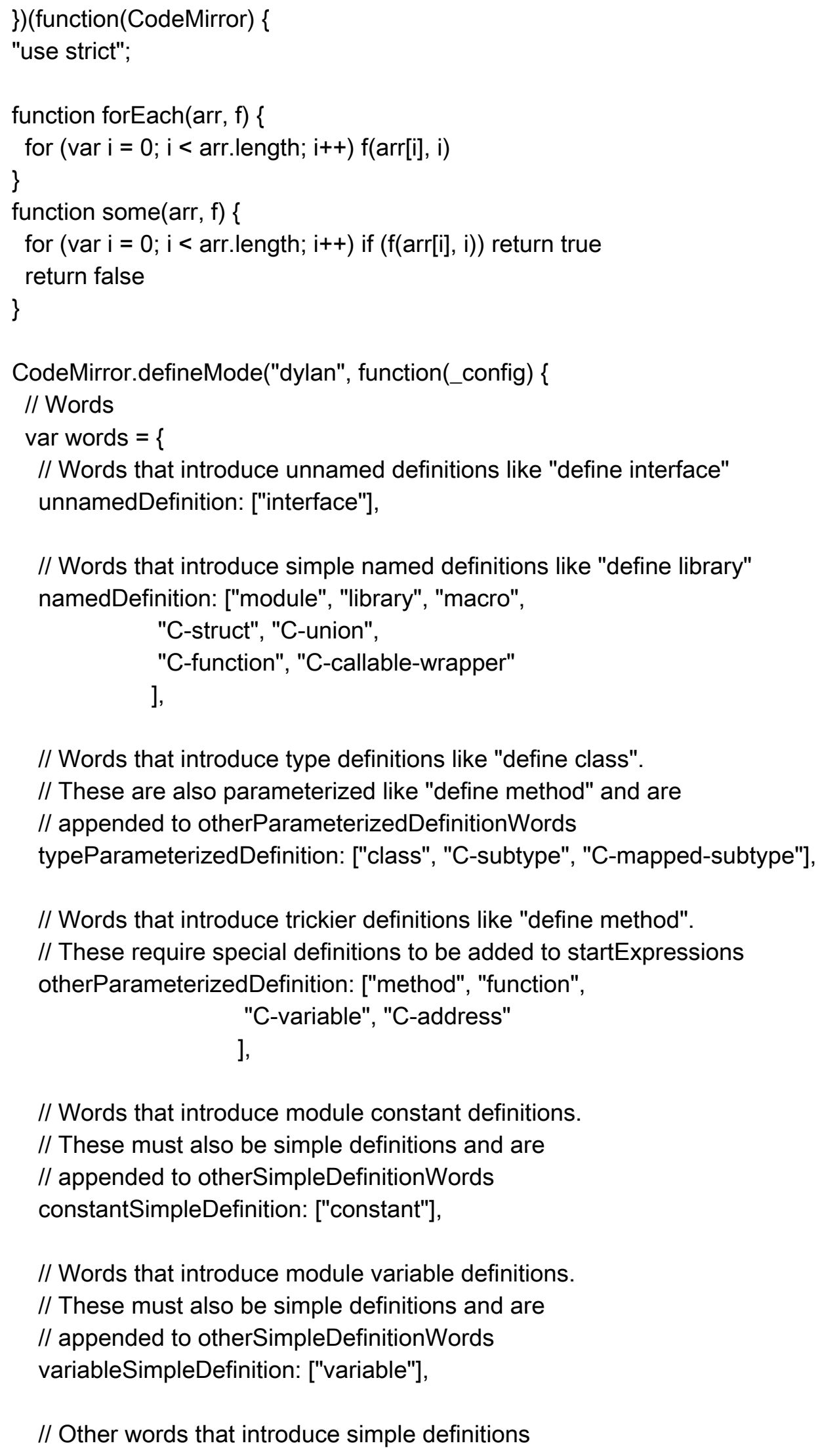

// Words that introduce type definitions like "define class".

// These are also parameterized like "define method" and are // appended to otherParameterizedDefinitionWords typeParameterizedDefinition: ["class", "C-subtype", "C-mapped-subtype"],

// Words that introduce trickier definitions like "define method".

// These require special definitions to be added to startExpressions otherParameterizedDefinition: ["method", "function",

"C-variable", "C-address"

],

// Words that introduce module constant definitions.

// These must also be simple definitions and are

// appended to otherSimpleDefinitionWords

constantSimpleDefinition: ["constant"],

// Words that introduce module variable definitions.

// These must also be simple definitions and are

// appended to otherSimpleDefinitionWords

variableSimpleDefinition: ["variable"],

// Other words that introduce simple definitions 
// (without implicit bodies).

otherSimpleDefinition: ["generic", "domain",

"C-pointer-type",

"table"

],

// Words that begin statements with implicit bodies.

statement: ["if", "block", "begin", "method", "case",

"for", "select", "when", "unless", "until",

"while", "iterate", "profiling", "dynamic-bind"

],

// Patterns that act as separators in compound statements.

// This may include any general pattern that must be indented

// specially.

separator: ["finally", "exception", "cleanup", "else", "elseif", "afterwards"

],

// Keywords that do not require special indentation handling,

// but which should be highlighted

other: ["above", "below", "by", "from", "handler", "in",

"instance", "let", "local", "otherwise", "slot",

"subclass", "then", "to", "keyed-by", "virtual"

],

// Condition signaling function calls

signalingCalls: ["signal", "error", "cerror",

"break", "check-type", "abort"

]

\}

words["otherDefinition"] =

words["unnamedDefinition"]

.concat(words["namedDefinition"])

.concat(words["otherParameterizedDefinition"]);

words["definition"] =

words["typeParameterizedDefinition"]

.concat(words["otherDefinition"]);

words["parameterizedDefinition"] =

words["typeParameterizedDefinition"]

.concat(words["otherParameterizedDefinition"]); 


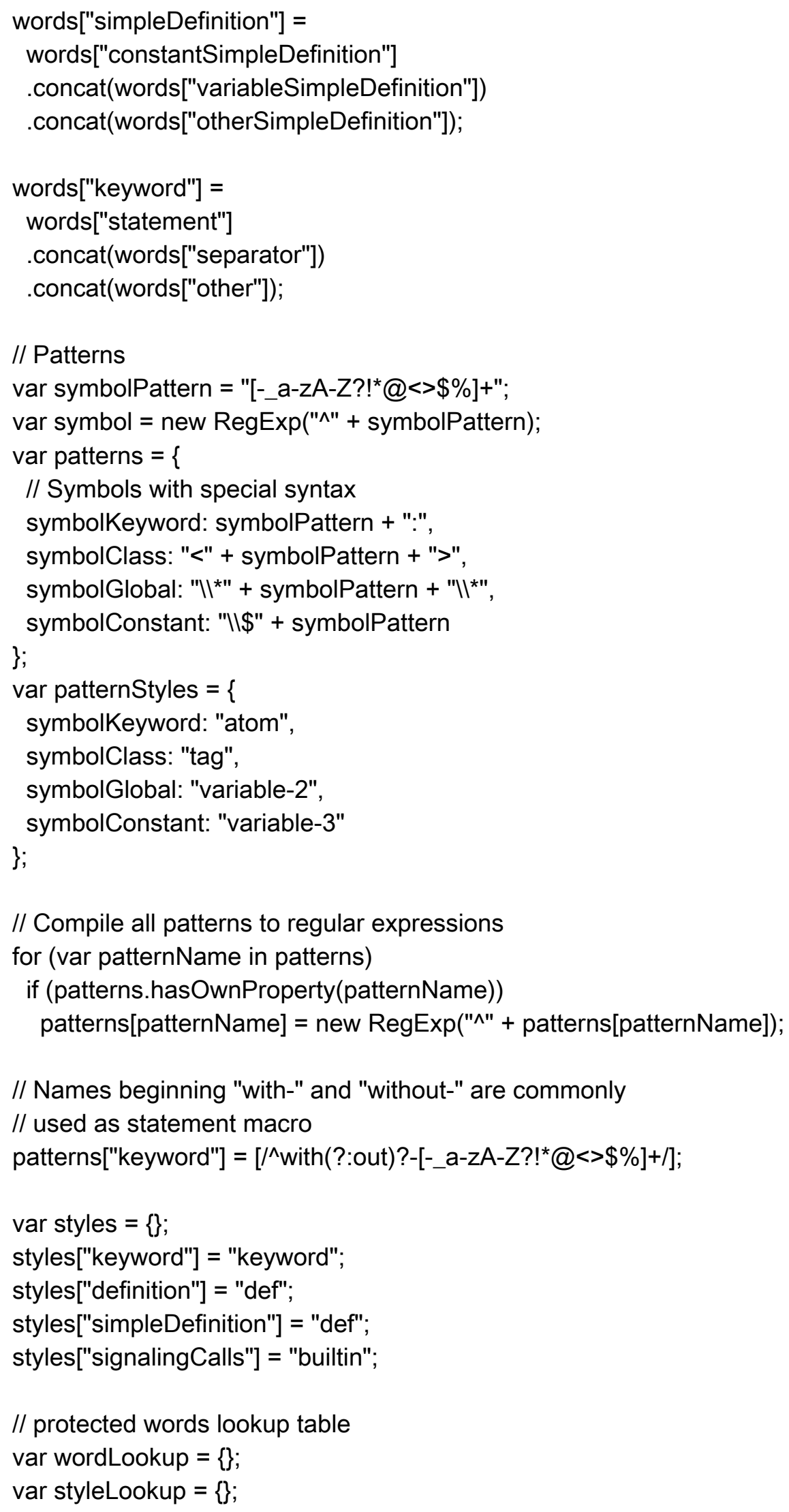




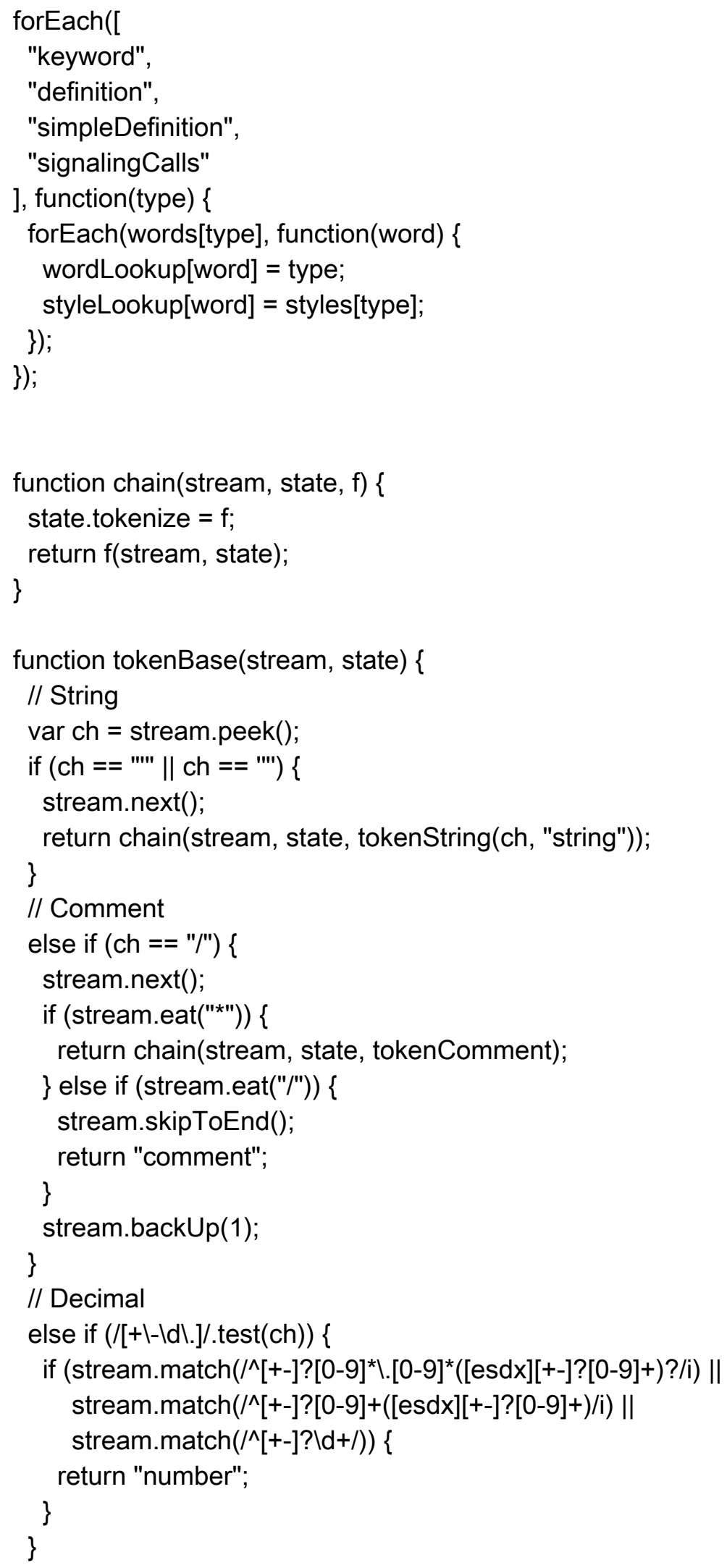




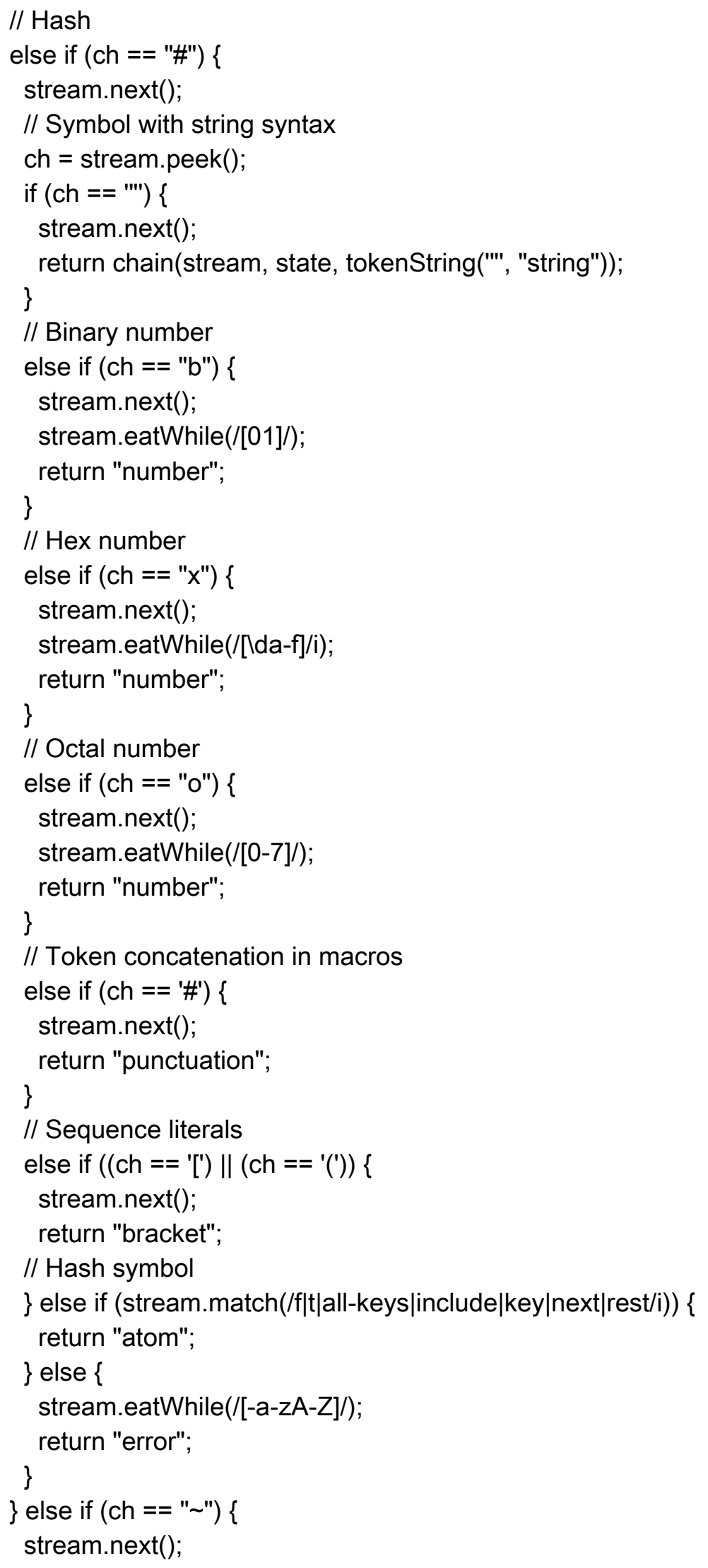




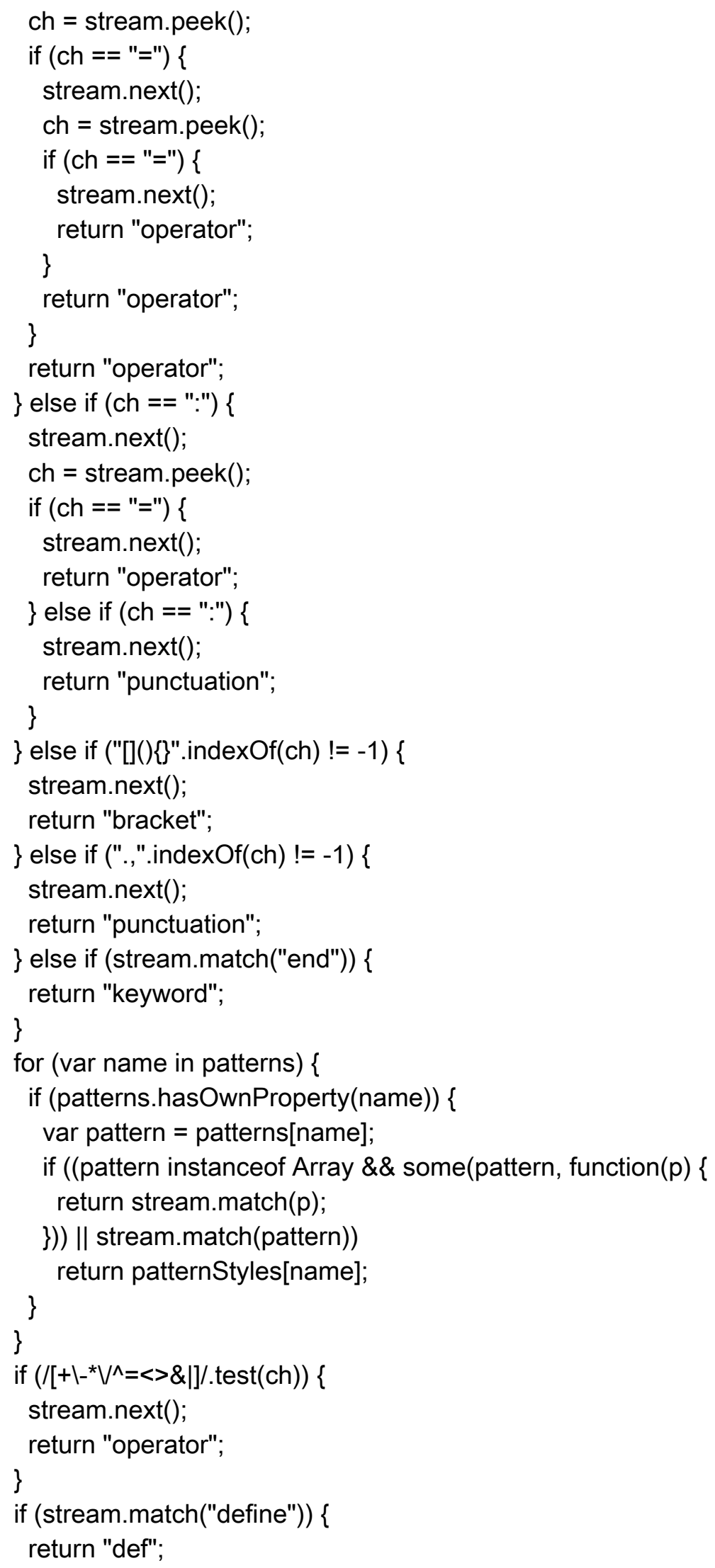




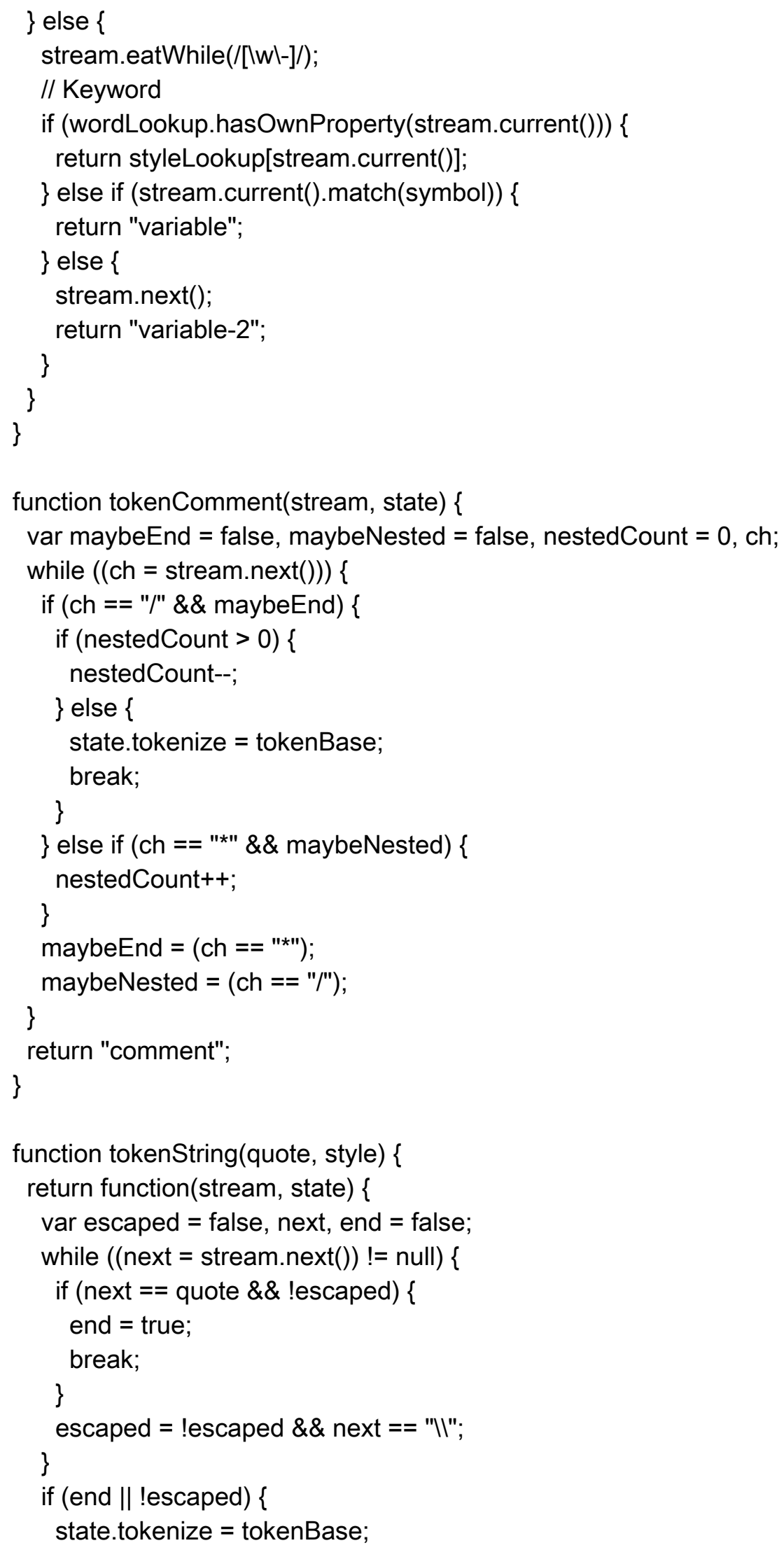




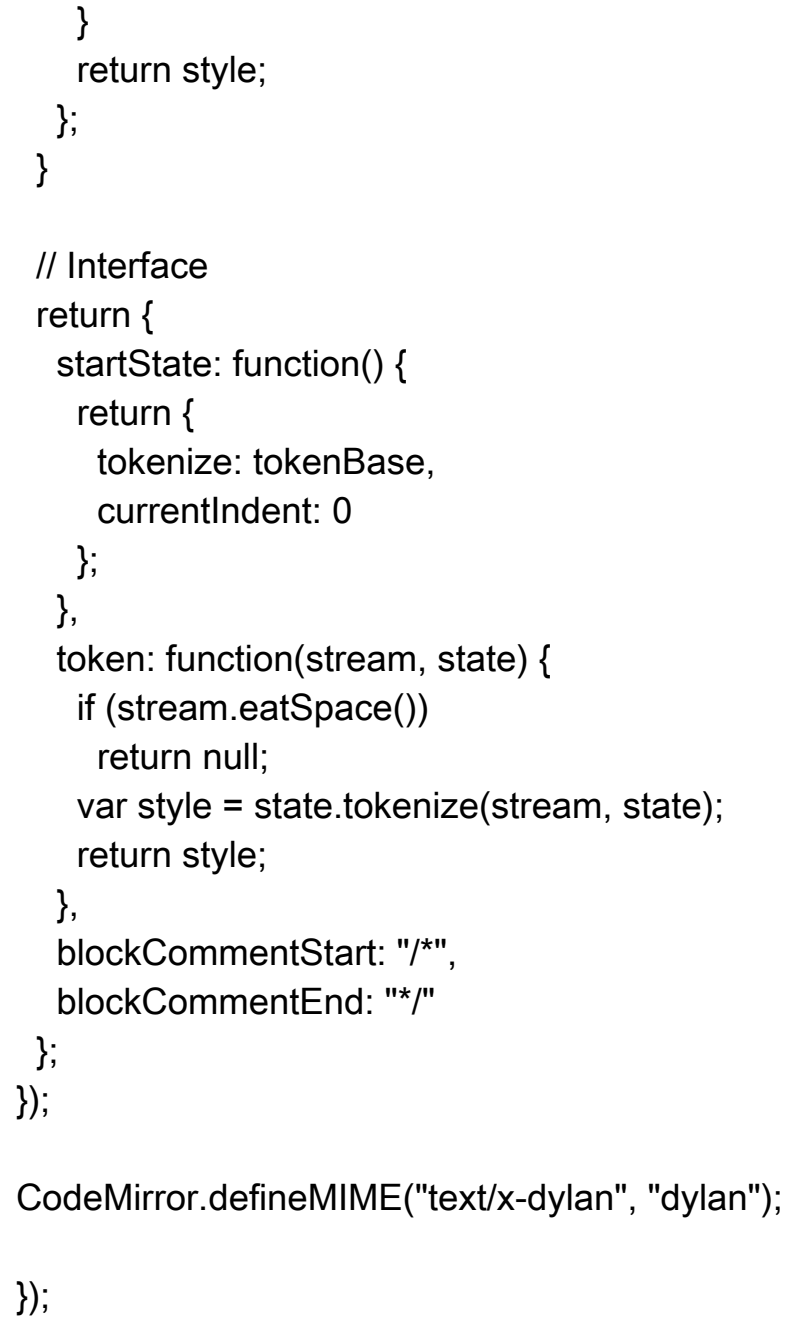

CodeMirror.defineMIME("text/x-dylan", "dylan");

\}); 